\title{
CONTROLLED-RELEASE PREDNISOLONE POLY (DL- LACTIDE) MICROSPHERES: IMPACT OF FORMULATION PARAMETERS, CHARACTERIZATION AND RELEASE MECHANISM
}

Khaled A. Khaled ${ }^{1}$, Hatem A. Sarhan ${ }^{1}$, Mohamed A. Ibrahim ${ }^{2}$ and Youssef W. Naguib ${ }^{1}$

${ }^{1}$ Department of Pharmaceutics, Faculty of Pharmacy, Minia University, Minia, Egypt

${ }^{2}$ Department of Pharmaceutics, Faculty of Pharmacy, Al-Azhar University, Assiut, Egypt

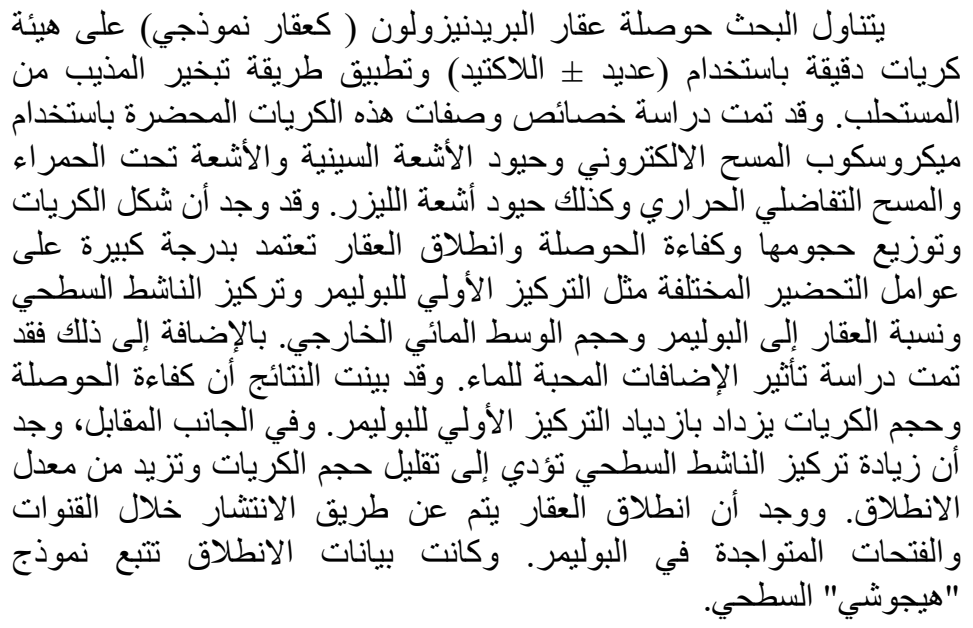

The steroidal drug prednisolone was encapsulated into microspheres using the biodegradable polymer poly (DL-lactide) using emulsion-solvent evaporation method. The produced microspheres were characterized using scanning electron microscopy, X-ray diffractometery, FT-IR spectroscopy, DSC, and laser light diffraction. The morphology, particle size distribution, encapsulation efficiency (EE\%), and drug release showed marked dependence upon formulation parameters viz. initial polymer concentration, surfactant concentration, drug-to-polymer ratio, and volume of the external aqueous phase. The effect of the addition of hydrophilic additives such as PVP or PEG 8000 was also 
investigated. The encapsulation efficiency percent and the mean particle size were increased by increasing the initial polymer concentration and drug polymer ratio. On the other hand, increasing the surfactant concentration resulted in decreasing the mean particle size and increasing the drug release from the microspheres. The probable mechanism of drug release was estimated and found to be via diffusion through channels and/or pores present within the polymeric matrix. Release data of almost all formulae fitted Higuchi's planar model better than spherical model. This finding could be due to the small extent of drug release $(\sim 40 \%)$, or the presence of a large fraction of the encapsulated drug nearby the surface of the microspheres.

\section{INTRODUCTION}

Poly lactide (PLA), glycolide (PGA), or lactide-co-glycolide (PLGA) based micro- and nanoparticles have been used to encapsulate various drug substances such as steroidal ${ }^{1}$ and non-steroidal anti-inflammatory drugs ${ }^{2}$, hormones ${ }^{3}$, and anti-cancer agents ${ }^{4}$, as well as various proteins and peptides ${ }^{5}$, and lately, live cells ${ }^{6}$. The advantages of using these biodegradable polymers and co-polymers are that they degrade to nontoxic monomers, thus, avoiding the need of surgical removal. In addition, the risk of long term toxicity or a probable immunological reaction when compared with non-degradable systems is minimized ${ }^{7}$. The degradation periods of these polymers may range from three weeks to over a year, depending on the composition of the copolymer, as well as the method of preparation and formulation ${ }^{8}$.

Small drug-loaded polymer particles may be called microcapsules, microspheres, nanoparticles, or pseudo-lattices ${ }^{9}$. Generally, parentral systems which are capable of being targeted to tissue sites and to deliver their active ingredient over a long period of time are of considerable interest ${ }^{10}$.

The encapsulation of steroidal anti-inflammatory agents into poly lactide or polylactide-co-glycolide polymers attracted the attention due to their wide applications. Eroglu et al. found that implantation of PLGAL-PLA microspheres containing dexa-methasone sodium phosphate is significantly more effective than the systemic administration of the drug in the treatment of brain edema ${ }^{11}$. Meanwhile Zolnik et al. carried out an elevated temperature accelerated release study on dexamethasoneloaded PLGA microspheres, and found a linear correlation between the accelerated-release and real-time data for erosion controlled systems. This elevated temperature studies appeared not to be suitable for PLGA systems where release was diffusioncontrolled $^{12}$. 
Prednisolone is a synthetic adrenal corticosteroid, very slightly soluble in water ${ }^{13}$. It has an antiinflammatory activity of 5 and salt retaining properties of 0.3 relative to hydrocortisone ${ }^{14}$. It is used to achieve prompt suppression of inflammation in many inflammatory conditions, as well as many allergic conditions. Chronic allergic and inflammatory conditions of the uvea, iris, conjunctiva and optic nerves of the eyes are also treated with prednisolone ${ }^{15}$. The drug oral bioavailability is $80 \%$. The $\mathrm{C}_{\max }$ is achieved after 1-3 hrs and $\mathrm{t}_{1 / 2}$ ranges between $2.5-3.5 \mathrm{hrs}^{16}$.

The objective of this study was to prepare sustained release prednisolone microspheres using the biodegradable polymer poly (DLlactide). The prepared microspheres may be suitable for the preparation of intramuscular injection. The drug seems to be a good candidate for the study, as it has a short half life, and given in small doses ${ }^{13}$. In this work, the impact of formulation parameters such as polymer concentration, surfactant concentration, drug: polymer ratio, the volume of the aqueous phase, and the additives, on the surface morphology, particle size distribution, encapsulation efficiency percent (EE\%), and drug release was investigated. It is worth mentioning that no previous similar report on prednisolone-loaded PLA microspheres was cited in the literature.

\section{EXPERIMENTAL}

\section{Materials}

Poly (DL-lactide) (PLA) (intrinsic viscosity of 0.55 to $0.75 \mathrm{dL} / \mathrm{g}$, molecular weight of 75-120 kd) was purchased from Aldrich (SigmaAldrich inc., St. Louis, MO, USA), Prednisolone (PD) was kindly granted from Al-Kahira Pharmaceutical Company (Cairo, Egypt), Polyvinyl alcohol (PVA) (partially hydrolyzed, degree of hydrolysis $88 \%$ ), and polyethylene glycol (PEG) 8000 were obtained from Fluka (Fluka Chemica, Germany), PVP (K30, molecular weight $40 \mathrm{kd}$ ) was obtained from Winlab (Winlab, Market Harborough, Leicestershire, UK). Other chemicals and reagents were of analytical grade and used as received.

\section{Methods}

\section{Preparation of the microspheres}

Microspheres were prepared using $\mathrm{o} / \mathrm{w}$ emulsion-solvent evaporation technique. Different weights of PLA was dissolved in $1.5 \mathrm{ml}$ of dichloromethane (DCM) in a screwcapped test tube to make solutions of $7.5-15 \% \mathrm{w} / \mathrm{v}$. Weighed amounts of prednisolone (PD) were then dispersed in the organic phase using a sonicated water bath (BranSonic 220, Zurich, Switzerland) for 10 minutes. The organic phase was then added drop by drop using a Pasteur pipette to $50 \mathrm{ml}$ of an aqueous PVA solution $(1,3$, or $5 \% \mathrm{w} / \mathrm{v})$ in a beaker stirred at $2000 \mathrm{rpm}$ by an over-head stirrer (Janke and Kenkel KG, Germany). Emulsification process continued for 10 minutes. The formed emulsion was then stirred under a slower speed (500 rpm) using a magnetic-type stirrer (Heidolph MR 3001, Heidolph, 
Germany) for 3 hours to evaporate the organic solvent. Microspheres were then harvested by centrifugation (Fischer Centrific ${ }^{\circledR}$ Centrifuge, USA) at $8000 \mathrm{rpm}$, washed 4 times with distilled water, and freeze-dried overnight (FreeZone 180, Labconco Corporation, Kansas city, Missouri, USA). Dried microspheres were kept in a desiccated bag and stored at $20^{\circ} \mathrm{C}$ pending investigation.

Additive-containing microspheres were prepared using the same procedure except that the additive (PVP or PEG) was dissolved in the organic phase prior to addition of the drug.

\section{Characterization of the micro- spheres \\ Morphology}

The morphology of the microspheres surfaces was investigated using scanning electron microscopy. Microspheres were spread on a carbon double-adhesive layer on a metal holder, and goldcoated using Ion-Sputtering device (Jeol Fine-Coat JFC 1100E, Jeol LTD, Tokyo, Japan). The microspheres were scanned by Scanning Electron Microscope (SEM) (Jeol JSM-5400 LV, Jeol LTD, Tokyo, Japan).

\section{Particle size analysis}

The size distribution of the PLA microspheres was investigated using laser light diffraction (Mastersizer 2000, Malvern Instruments, Herrenberg, Germany). For a typical experiment, about $10 \mathrm{mg}$ of microspheres was suspended in $5 \mathrm{ml}$ water and analyzed. All samples were analyzed 5 times and average results were taken. The sizes below which $10 \%(\mathrm{~d}(0.1)), 50 \%(\mathrm{~d}(0.5))$ and $90 \%$ $(d(0.9))$ of the microspheres fell were used to characterize the microsphere size distribution. The mean diameter was taken as the average of $\mathrm{d}(0.1)$, $\mathrm{d}(0.5)$, and $\mathrm{d}(0.9)$ values.

\section{Encapsulation efficiency}

Weighed amounts of the microspheres were dispersed in a small volume of DCM to dissolve the polymer. A volume of $50 \mathrm{ml}$ of phosphate buffer saline (PBS) pH 7.4 was then added, the mixture was stirred using the magnetic stirrer to evaporate DCM. The remaining aqueous medium was centrifuged, and aliquots of the supernatant were analyzed for the drug using a UV/Vis spectrophotometer (Jenway 6505 UV/VIS spectrophotometer, Jenway LTD, Essex, UK) at $245 \mathrm{~nm}$. Drugfree microspheres were prepared and subjected to the same procedure, and the supernatant obtained from which was used as a blank. It's worth mentioning that the used additives, including the polymer were found not to interfere with the method of assay, as the maximum absorbance wavelength, and absorptivity were found to be unaffected by these additives. Experiments were carried out in triplicate and the average values were reported.

Drug encapsulation efficiency percent (EE\%) was calculated using the following formula: 
$\mathrm{EE} \%=\frac{\text { actual amount of drug in microspheres }}{\text { theoreticd amount of drug in microspheres }} \times \mathbf{1 0 0}$

\section{Release study}

Approximately $10 \mathrm{mg}$ of PDloaded microspheres were suspended in $6 \mathrm{ml}$ PBS in screw-capped test tubes. The tubes were kept under constant shaking $(60 \mathrm{rpm})$ in a shaking water bath (PolyScience, model 20 L-M, Niles, IL, USA) at $37^{\circ} \mathrm{C}$. The release experiments were carried out under sink condition, where the drug concentration in the release medium was not exceeding $10 \%$ of the saturation concentration. It is important to mention that the saturation solubility of the drug in the buffer used at $37^{\circ} \mathrm{C}$ was measured in our lab and found to be $0.38 \mathrm{mg} / \mathrm{ml}$, and the concentration of the drug in the release medium at different time intervals of the whole experiment did not exceed $0.03 \mathrm{mg} / \mathrm{ml}$. At time intervals, the tubes were centrifuged, and $5 \mathrm{ml}$ were withdrawn from each tube and replaced with $5 \mathrm{ml}$ of fresh buffer (kept at the same temperature). The drug concentration was determined spectrphotometrically at 245 $\mathrm{nm}$ in the withdrawn samples. Release experiments were conducted in triplicate for each batch.

\section{X-Ray diffraction}

The X-ray diffractograms were obtained using Jeol XR Diffractometer (Jeol, Tokyo, Japan). The radiation source was a copper $(\lambda=1.54184 \AA)$ high-intensity x-ray tube operated at $35 \mathrm{KV}$ and a current of $15 \mathrm{~mA}$. The diffraction patterns were achieved using continuous scan mode with $2 \theta$ values ranging from 4100 at a rate of 4 degrees/minute.

\section{Differential scanning calorimetry (DSC)}

The DSC patterns were obtained using a differential scanning calorimeter DSC (Perkin Elmer, 2-C, NY, USA) with a thermal analysis data station system, by heating $5 \mathrm{mg}$ samples from $30-300^{\circ} \mathrm{C}$ at a scanning rate of $5^{\circ} \mathrm{C} / \mathrm{min}$ in sealed aluminum pans, under a stream of nitrogen gas at flow rate of $40 \mathrm{ml} / \mathrm{min}$. The instrument was calibrated using an indium standard.

\section{Fourier-Transform Infrared Spectroscopy (FT-IR)}

FT-IR Spectroscopy was carried out using potassium bromide disk method. Samples, 1-2 mg each, were mixed with potassium bromide, compressed at a pressure of $6 \mathrm{ton} / \mathrm{cm}^{2}$ into discs and scanned using a Perkin Elmer FT-IR spectrophotometer (Perkin Elmer, NY, USA) over the range of $4000-500 \mathrm{~cm}^{-1}$. A blank $\mathrm{KBr}$ pellet was used as a reference.

\section{RESULTS AND DISCUSSION}

Among the different methods employed to prepare polymeric drugloaded microspheres, the method of emulsion solvent evaporation was applied. This method is suitable for encapsulating lipophilic drugs that can be either dispersed or dissolved in 
the oily phase (the volatile solvent) ${ }^{7}$. The influence of different formulation parameters of the used method was investigated, along with the effect of additives. Table 1 shows different formulae with their formulation parameters. It is worth mentioning that the whole batches of all formulations were used in the in vitro and physico-chemical characterization studies without further fractionation.

Table 1: Formulation parameters and properties of different formulae of Prednisolone microspheres.

\begin{tabular}{|c|c|c|c|c|c|c|c|c||}
\hline F\# & $\begin{array}{c}\text { PLA } \\
\% \mathrm{w} / \mathrm{v}\end{array}$ & $\begin{array}{c}\text { PVA } \\
\% \mathrm{w} / \mathrm{v}\end{array}$ & $\begin{array}{c}\text { D:P } \\
\text { ratio }\end{array}$ & $\begin{array}{c}\text { O/W } \\
\text { ratio }\end{array}$ & $\begin{array}{c}\text { additives } \\
(\% \mathrm{w} / \mathrm{w})\end{array}$ & $\begin{array}{c}\text { Yield } \\
\%\end{array}$ & $\%$ content $\pm \mathrm{SD}^{\mathrm{a}}$ & $\begin{array}{c}\text { EE\% } \pm \text { SD } \\
\text { a,b }\end{array}$ \\
\hline 1 & 7.5 & 1 & $01: 04$ & $1.5: 50$ & - & 57.35 & $5.07 \pm 0.50$ & $25.35 \pm 3.07$ \\
\hline 2 & 10 & 1 & $01: 04$ & $1.5: 50$ & - & 62.46 & $10.85 \pm 0.45$ & $54.25 \pm 2.27$ \\
\hline 3 & 12.5 & 1 & $01: 04$ & $1.5: 50$ & - & 62.21 & $13.6 \pm 0.28$ & $67.96 \pm 1.42$ \\
\hline 4 & 12.5 & 3 & $01: 04$ & $1.5: 50$ & - & 76.03 & $13.69 \pm 0.13$ & $68.43 \pm 0.64$ \\
\hline 5 & 12.5 & 5 & $01: 04$ & $1.5: 50$ & - & 67.18 & $10.84 \pm 0.77$ & $54.2 \pm 3.85$ \\
\hline 6 & 12.5 & 1 & $01: 06$ & $1.5: 50$ & - & 57.05 & $7.96 \pm 0.42$ & $55.71 \pm 2.97$ \\
\hline 7 & 12.5 & 1 & $01: 03$ & $1.5: 50$ & - & 68.26 & $23.3 \pm 0.12$ & $93.25 \pm 0.47$ \\
\hline 8 & 12.5 & 1 & $01: 02$ & $1.5: 50$ & - & 56.76 & $32.45 \pm 1.57$ & $97.35 \pm 4.70$ \\
\hline 9 & 12.5 & 1 & $01: 04$ & $1.5: 100$ & - & 54.41 & $15.47 \pm 0.31$ & $77.34 \pm 1.56$ \\
\hline 10 & 12.5 & 1 & $01: 04$ & $1.5: 200$ & - & 50.28 & $13.65 \pm 0.46$ & $68.25 \pm 2.31$ \\
\hline 11 & 12.5 & 1 & $01: 04$ & $1.5: 50$ & 7.5 PEG & 34.07 & $13.0 \pm 0.39$ & $65.10 \pm 1.98$ \\
\hline 12 & 12.5 & 1 & $01: 04$ & $1.5: 50$ & 15 PEG & 20.9 & $11.32 \pm 1.15$ & $56.60 \pm 5.54$ \\
\hline 13 & 12.5 & 1 & $01: 04$ & $1.5: 50$ & 25 PEG & 12.96 & $9.17 \pm 0.58$ & $45.90 \pm 2.89$ \\
\hline 14 & 12.5 & 1 & $01: 04$ & $1.5: 50$ & 2 PVP & 67.92 & $7.07 \pm 0.41$ & $35.35 \pm 2.05$ \\
\hline 15 & 15 & 5 & $01: 04$ & $1.5: 50$ & & 79.96 & $12.5 \pm 0.20$ & $62.60 \pm 0.99$ \\
\hline 16 & 15 & 5 & $01: 04$ & $1.5: 50$ & 0.5 PVP & 66.74 & $13.77 \pm 0.69$ & $68.87 \pm 3.45$ \\
\hline 17 & 15 & 5 & $01: 04$ & $1.5: 50$ & 0.1 PVP & 68.22 & $11.24 \pm 0.33$ & $56.2 \pm 1.65$ \\
\hline
\end{tabular}

${ }^{\mathrm{a}}$ calculated as the average value $\pm \mathrm{SD}(\mathrm{n}=3)$. ${ }^{\mathrm{b}} \mathrm{EE} \%$ means encapsulation efficiency percent. 


\section{Microspheres morphology}

SEM images of the formulae show that almost all the microparticles are spherical in shape. Microspheres with low drug loading and no additives (such as formula F\#3 and F\#15, Fig. 1a and $1 \mathrm{~b}$ ) showed smooth and intact surfaces, with few small drug crystals scattered on the surface. On the other hand, those with higher drug loading (e.g. formula F\#8, Fig. 1c) showed corrugated surfaces due to the presence of the drug crystals interspersed with the smooth surface of the polymer. Upon the addition of various concentrations of PEG 8000 $(7.5,15,25 \% \mathrm{w} / \mathrm{w})$ to formula $\mathrm{F} \# 3$; formula F\#11, F\#12, F\#13 were produced, respectively. Generally, the addition of PEG increased the porosity of the microspheres surfaces, (F\#13, Fig. 1d). On the other hand, PVP was added in a concentration of $2 \% \mathrm{w} / \mathrm{w}$ to formula $\mathrm{F} \# 3$ to produce formula F\#14, characterized by high deposition of drug crystals on the surface of the microspheres (Fig. 1e).

\section{Size of the microspheres}

Size distribution of formula F\#2 is shown in Figure 2. Generally, the size of the microspheres showed dependence on a variety of parameters. Upon changing the PLA concentration in the organic phase from 7.5, to 10 , then to $12.5 \% \mathrm{w} / \mathrm{v}$ (formulae $\mathrm{F} \# 1, \mathrm{F \# 2}$, and F\#3 respectively), the particle size increased (Table 2). It is postulated that the higher polymer concentrations resulted in a more viscous oily phase, which exerted more viscous resistance during the emulsification process against the rotation of the paddle, forming larger particles ${ }^{17}$. On the contrary, the mean particle size decreased when higher surfactant concentrations were used (F\#3, 4 and 5, Table 2).

Table 2: Size distribution data of different formulae of prednisolone microspheres

\begin{tabular}{|c|c|c|c|c|c||}
\hline \hline F\# & $\begin{array}{c}\mathrm{d}(0.1) \\
(\text { um })\end{array}$ & $\begin{array}{c}\mathrm{d}(0.5) \\
(\mathrm{um})\end{array}$ & $\begin{array}{c}\mathrm{d}(0.9) \\
(\mathrm{um})\end{array}$ & $\begin{array}{c}\text { Mean diameter } \\
(\mathrm{um})\end{array}$ & Uniformity \\
\hline 1 & 22.3 & 52.4 & 86.7 & 53.8 & 0.37 \\
\hline 2 & 34.3 & 76.0 & 124.9 & 78.4 & 0.39 \\
\hline 3 & 63 & 108.3 & 160.7 & 110.6 & 0.35 \\
\hline 4 & 40.1 & 68.9 & 128.8 & 79.3 & 0.55 \\
\hline 5 & 17.5 & 36.5 & 73.6 & 42.5 & 0.5 \\
\hline 6 & 51.258 & 89.375 & 120.316 & 86.7 & 0.235 \\
\hline 7 & 65.047 & 110.185 & 269.908 & 148.38 & 0.576 \\
\hline 8 & 74.467 & 133.315 & 380.552 & 196.1 & 0.64 \\
\hline 9 & 81 & 116.5 & 159 & 118.8 & 0.21 \\
\hline 10 & 65.6 & 110.7 & 230.5 & 136 & 0.49 \\
\hline
\end{tabular}




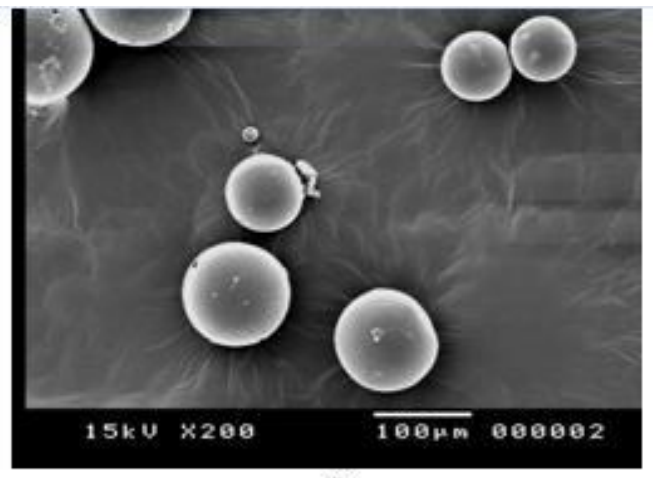

a

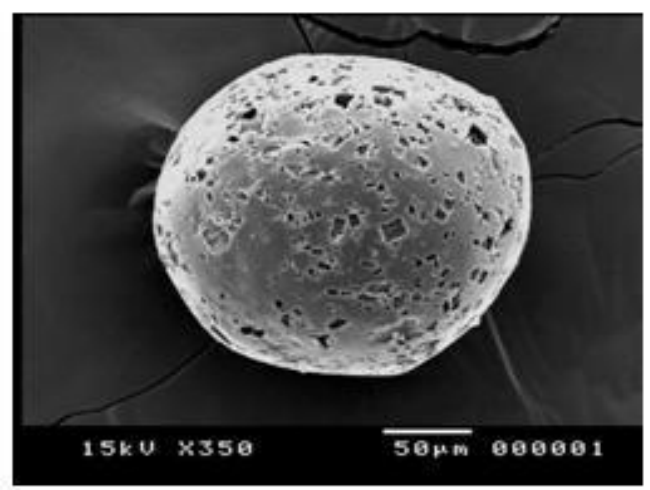

C

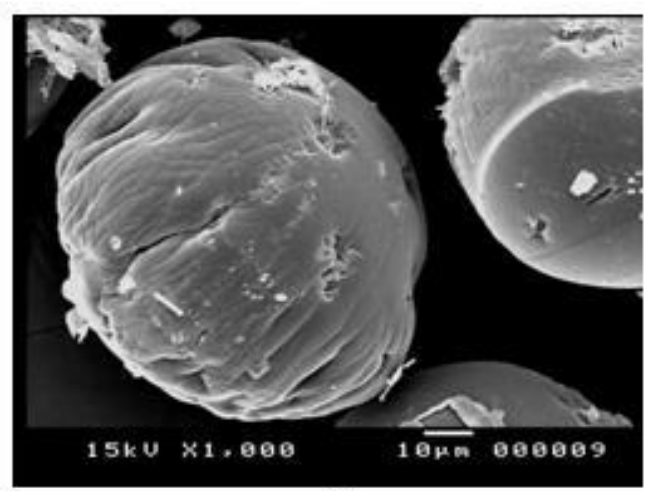

e

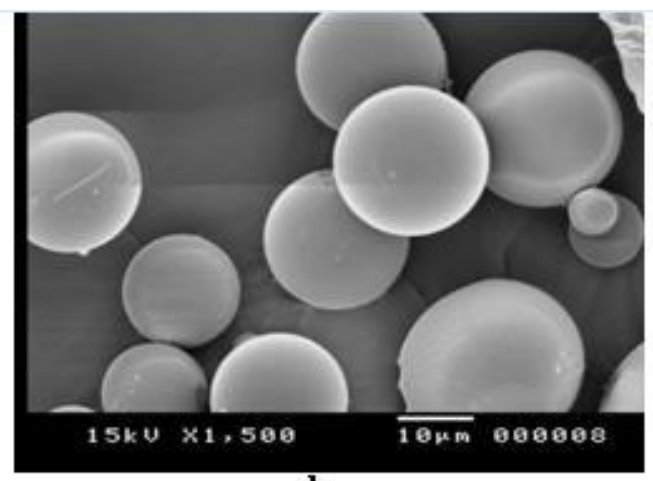

b

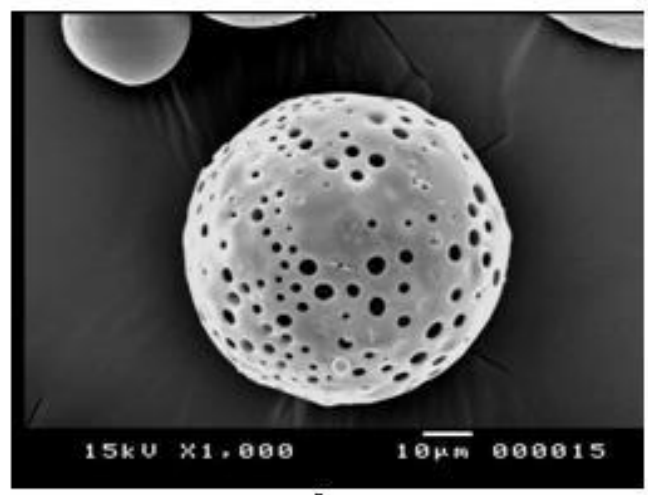

d

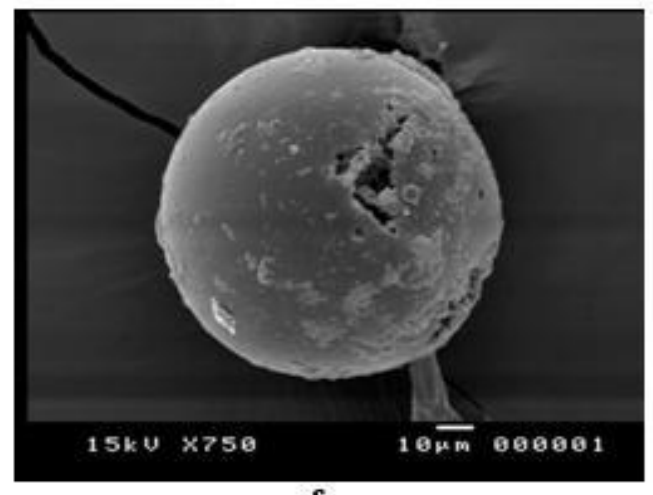

f

Fig. 1: Scanning electron micrographs of the prepared microspheres: (a) F\#3, (b) F\#15, (c) F\#8, (d) F\#13, (e) F\#14, (f) F\#3 after 28 days of release experiment. 


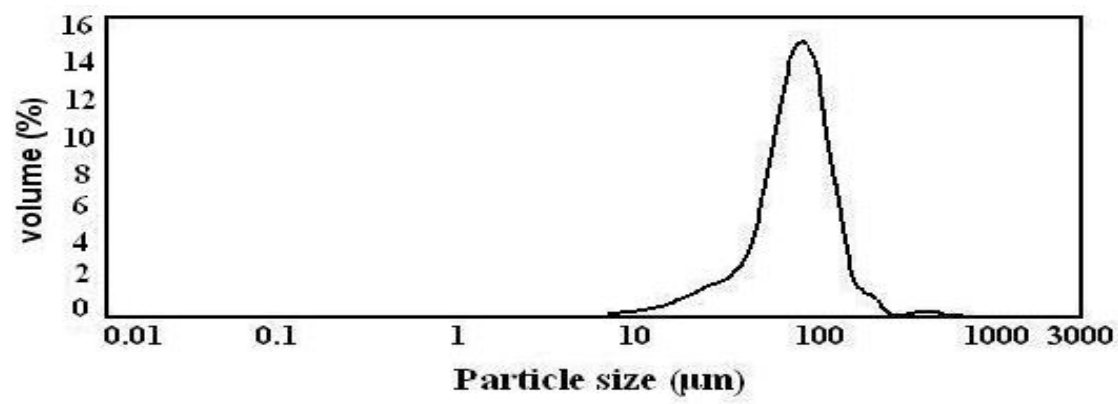

Fig. 2: Particle size distribution of prednisolone-loaded PLA microspheres (F\#2).

It was also found that producing microspheres with different drug:PLA ratios (formulae F\#3, F\#6, F\#7, and F\#8 with drug:PLA ratios of 1:4, 1:6, $1: 3$, and $1: 2$ respectively) resulted in changing the mean particle sizes in a direct proportion to the drug:PLA ratio (Table 2 ).

The volume of the external aqueous phase plays an important role on the emulsion stability, as well as the rate of solvent removal from the microspheres, and thus, the time required for their solidification. It was reported that increasing the w:o ratio enhances the rate of solvent removal $^{18}$. Polymer precipitation at earlier stages prevents further shrinkage of the microspheres, therefore, the increase in o: $\mathrm{W}$ ratio from $50 \mathrm{ml}$, to $100 \mathrm{ml}$, and to $200 \mathrm{ml}(\mathrm{F} \# 3, \mathrm{~F} \#$, and $\mathrm{F} \# 10$ respectively) results in an increase in the mean particle size of the produced batches (Table 2).

\section{Yield of the produced microspheres}

It is important to mention that hollow spheres were formed during preparation of the microspheres, they were found floating on the external aqueous phase by the end of the process. These hollow spheres were separated and removed from the preparation. This may explain the low yield values that range from about 55 to $75 \%$. The smallest yield values were obtained upon using PEG as an additive. Similar results were reported when hydroxypropyl $\beta$-cyclodextrin was used as an additive ${ }^{19}$.

It was observed that using higher surfactant concentrations (formulae $\mathrm{F} \# 3$, F\#4, and F\#5) increased the stability of the formed emulsions, and thus, decreased the amount of the hollow spheres formed, resulting in higher yield values (Table 1). Same observation was also noticed in formulae $\mathrm{F} \# 15, \mathrm{~F} \# 16$, and $\mathrm{F} \# 17$ (Table 1).

Increasing in the external aqueous phase volume to $100 \mathrm{ml}$, or $200 \mathrm{ml}$ (formulae $\mathrm{F} \# 9$, and $\mathrm{F} \# 10$ respectively) gave smaller yield values, compared to F\#3 (Table 1). It is assumed that decreasing oil:water ratio resulted in decreased the emulsion stability, resulting in the formation of larger number of hollow spheres.

Encapsulation efficiency and
release profiles


To study the effect of polymer concentration on $\mathrm{EE} \%$ and release, three polymer concentrations were used: $7.5 \%, 10 \%$, and $12.5 \% \mathrm{w} / \mathrm{v}$, represented in formulae $\mathrm{F} \# 1, \mathrm{~F} \# 2$, and F\#3, respectively. The results are shown in Figure 3. It was found that as the polymer concentration increased, the $\mathrm{EE} \%$ was also increased. This may be attributed to the increased viscosity of the organic phase upon using high polymer concentrations. The increase of the

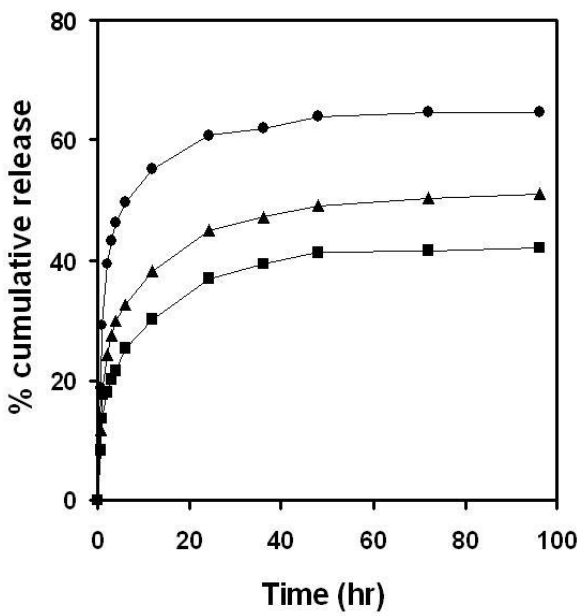

Fig. 3: Effect of PLA concentration on the release of prednisolone from: F\#3 (PLA 12.5\% w/v) (घ), F\#2 (PLA 10\% w/v) ( $\mathbf{\Delta})$, and F\#1 (PLA 7.5\% w/v) (•).

viscosity resulted in increasing the resistance which faced the drug during its diffusion to the external aqueous phase (i.e. decreasing drug permeability). In addition, the time required for the microspheres to solidify decreased upon using high polymer concentrations. Hence, the drug had shorter time to escape to the external phase. Moreover, the use of high polymer concentrations was found to increase the mean diameter of the microspheres which leads to a longer diffusion pathway. Similar results were found in the literature ${ }^{20}$.

The amounts released of the drug from these three formulae $(\mathrm{F} \# 1, \mathrm{~F} \# 2$, and F\#3) were decreased by increasing the polymer concentration from $49.5 \%$ to $25.3 \%$ during the first 6 hours of the experiment. Similarly, the cumulative amounts released during the whole period of the experiment were decreased from $64.6 \%$ to $42.1 \%$. This finding could be due to the enlargement of the microspheres mean size, resulting in decreasing the surface area exposed to the release medium. In addition, it is also suggested that this release retardation may be due to the formation of a denser microsphere structure when higher PLA concentrations were used during the preparation $^{21}$. It is important to notice that the microspheres did not show complete release of the encapsulated drug, and the release rate was markedly decreasing with time (Fig. 3 ), suggesting that complete drug release will take very long time ${ }^{22}$ due to the high molecular weight of the used polymer which will result in very slow degradation rates. This explanation is supported by an SEM image of a microsphere from formula F\#3 which shows a small degree of erosion after 28 days of incubation (Fig. 1f).

The influence of surfactant concentration was investigated using three PVA concentrations: 1\%, 3\%, and $5 \% \mathrm{w} / \mathrm{v}$ of PVA, (formulae F\#3, $\mathrm{F} \# 4$, and $\mathrm{F \# 5}$ respectively). Table 1 
reveals that increasing the surfactant concentration from 1 to $3 \%$ did not seem to have a significant impact on the $\mathrm{EE} \%$. However, further increase of the PVA concentration to 5\% lowered the EE\% to $54.2 \%$ in formula $\mathrm{F} \# 5$ (compared to $68 \%$ and $68.4 \%$ in formulae F\#3 and F\#4, respectively).

Meanwhile, the reduction in the mean size due to increasing PVA concentration gave its effect on the drug release. Smaller particles (F\#5) released $42 \%$ of the encapsulated drug during the first 6 hours, and $57.1 \%$ at the end of the experiment, compared to $25.3 \%$ and $42.1 \%$ for larger microspheres (F\#3) as illustrated in Figure 4.

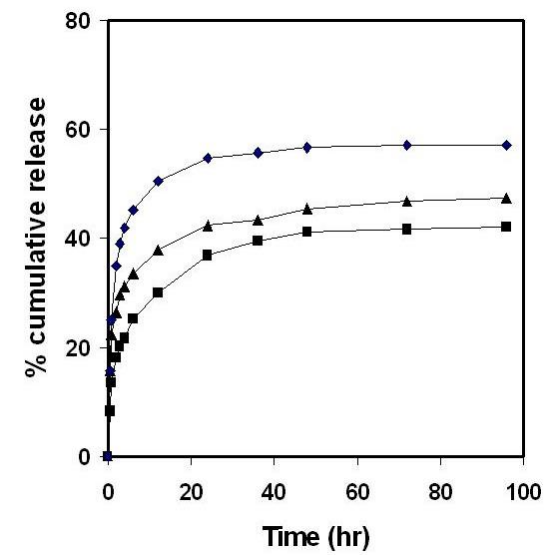

Fig. 4: Effect of PVA concentration on the release of prednisolone from: F\#3 (PVA 1\% w/v) (匹), F\#4 (PVA 3\% w/v) (A), and F\#5 (PVA 5\% w/v) (»).

Table 1 shows that increasing the drug:PLA ratio from $1: 6$ to $1: 4$ (formulae $\mathrm{F} \# 6$ and $\mathrm{F} \# 3$ ) increased EE\% from $55.7 \%$ to $68 \%$. Further increase in the ratio to $1: 3$ (formula $\mathrm{F} \# 7)$ gave $\mathrm{EE} \%$ of $93.3 \%$. The highest EE\% value (97.4\%) was obtained when the ratio was increased to 1:2 (formula F\#8). This can be explained by the fact that PD has a slight solubility in the external aqueous phase. Upon increasing the initial drug content, the amount that escapes to the external aqueous phase did not significantly change, and thus, the amount that remains in the oily phase, awaiting polymer coating, increases.

Increasing drug:PLA ratio increased the amount released of the drug (Fig. 5). The amount released during the first 6 hours increased from $11.9 \%$ (formula $F \# 6$ ) to $36.6 \%$

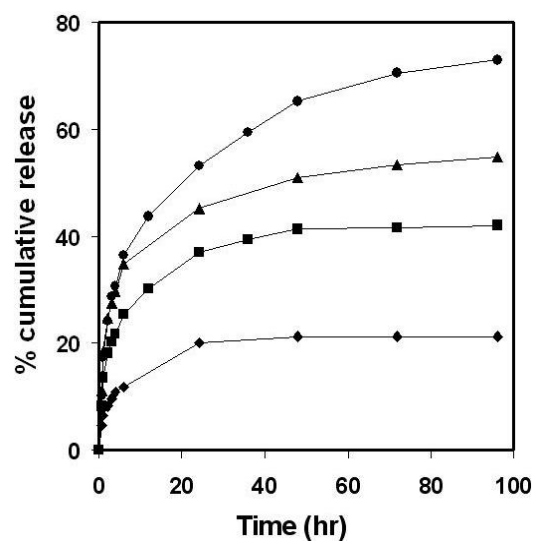

Fig. 5: Effect of drug:PLA ratio on the release of prednisolone from: F\#6 (drug:PLA ratio 1:6) (४), F\#3 (drug:PLA ratio 1:4) (๘), F\#7 (drug:PLA ratio 1:3) ( $\mathbf{\Delta})$, and $\mathrm{F} \# 8$ (drug:PLA ratio 1:2)

$(\bullet)$.

(formula F\#8). The cumulative amounts released by the end of the experiment were also increased from $21.2 \%$ (formula F\#6) to $72.9 \%$ (formula F\#8). The corrugated surfaces of microspheres of formula F\#8 (Fig. 1c) were observed to contain drug crystals embedded 
within and underneath the polymer matrix. This can lead to a continual increase in porosity of the polymer as more drug is released, and allow release of larger amounts of the drug encapsulated deep inside the polymer matrix. Similar explanation was also reported $^{23}$.

The effect of external phase volume on drug $\mathrm{EE} \%$ can be attributed to two opposite factors. The first one is the fast microspheres solidification due to the increased external phase volume which led to solvent extraction in addition to solvent evaporation ${ }^{18 \& 20}$. This phenomenon tends to increase drug $\mathrm{EE} \%$, because drug molecules are entrapped within the rapidlysolidified polymer. The second factor is the increase in the drug amount that escapes to the external aqueous phase upon increasing the external phase volume, resulting in a decrease in drug EE\%. These two opposite factors seem to outweigh each other as shown in Table 1. As was previously mentioned, the larger volume enabled the microspheres to solidify faster, producing larger microspheres (Table 2), with smaller surface areas subjected to the release medium. This explains the smaller release of the drug (Fig. 6). During
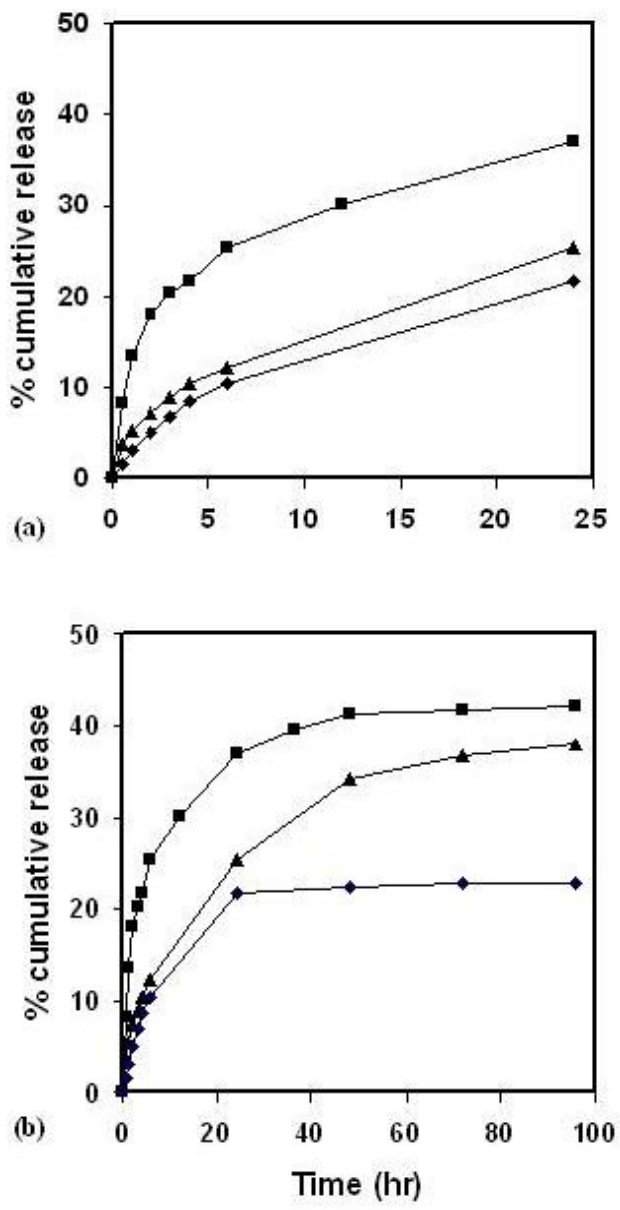

Fig. 6: Effect of external phase volume on the release of prednisolone from: F\#10 (200 ml) ( $), F \# 9$ $(100 \mathrm{ml})(\boldsymbol{\Delta})$, and $\mathrm{F} \# 3(50 \mathrm{ml})$ (घ). (a) for 24 hours, (b) for 96 hours.

the first 24 hours, formula $\mathrm{F} \# 3$ released $37 \%$ of the encapsulated drug, meanwhile, formulae F\#9 and F\#10 released $25.3 \%$ and $21.7 \%$ respectively during the same period. It is believed that this retardation in drug release may also be attributed to the decrease in the drug amounts 
present on or close to the surface when the volume of the external phase was increased. The drug is thought to migrate to the surface during the slow process of solvent evaporation, so that, the fast microspheres solidification during solvent evaporation inhibits further drug migration to the surface, resulting in the release of only small fractions of the incorporated drug during the period of the release experiment.

With regard to the influence of additives, PEG 8000 was used as a channeling agent to increase the porosity of microspheres. The PEGcontaining microspheres appeared highly perforated (Fig. 1d). Table 1 illustrates that the EE\% decreased upon increasing the PEG \%. The use of channeling agents provided larger surface areas to the release medium, hence, increasing the PEG 8000 percent in the microspheres (from $7.5 \%$ to $15 \%$, and $25 \% \mathrm{w} / \mathrm{w}$ in

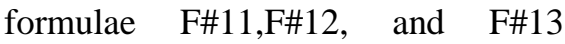
respectively) enhanced the burst release, as well as increased the cumulative amounts of drug released (Fig. 7).

The effect of addition of PVP (K30) was quite different. On the contrary of what was reported ${ }^{24}$. PVP did not behave as a channeling agent. The release of the drug from formula F\#14 (2\% w/w PVP) was very rapid, liberating about $87.53 \%$ of the "encapsulated" drug in the first 6 hours. Upon observing the microspheres of formula F\#14 under the light microscope (results not shown), there was a clear external layer of the drug covering the microspheres. It is suggested that the addition of PVP enhanced the water solubility of the drug and increased its affinity towards the external aqueous phase, resulting in accumulation of the drug crystals onto or near the surface of the microspheres. The solubility of the drug in an aqueous phase containing $2 \%$ w/w of PVP was determined experimentally in our laboratory and found to be approximately $32 \%$ higher than the solubility of the drug in the aqueous phase free from PVP. Of course the amount of the drug lost during washing was great, resulting in low $\mathrm{EE} \%$ of only $35.4 \%$. instead of $68 \%$ in the additive-free formula (F\#3). SEM images show clearly that the drug crystals "cover" the microspheres (Fig. 1e).

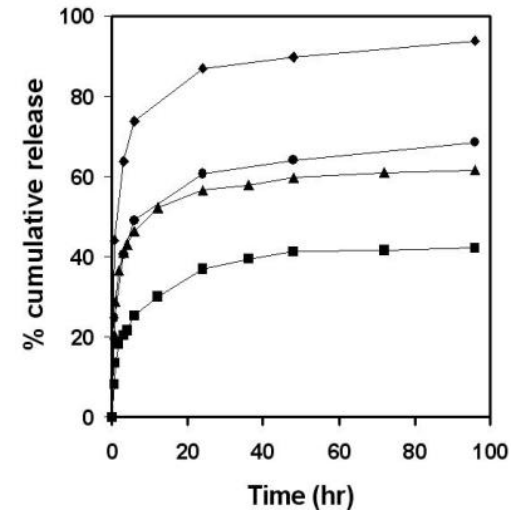

Fig. 7: Effect of the concentration of PEG 8000 on the release of prednisolone from: F\#3 (no additive) (ロ), F\#11 (PEG 7.5\% $\mathrm{w} / \mathrm{w})(\boldsymbol{\Delta}), \quad \mathrm{F} \# 12$ (PEG $15 \%$ $\mathrm{w} / \mathrm{w})(\bullet$ ), and F\#13 (PEG 25\% $\mathrm{w} / \mathrm{w})(\diamond)$.

\section{Solid state characterization}

Formula \#3 was used in the solid state characterization study, in 
addition to the drug free microspheres, and drug alone. X-ray diffraction patterns show the crystalline peaks of the pure drug (Fig. 8), as well as the amorphous pattern characteristic of the polymer. On the other hand, x-ray diffractograms of drug-loaded microspheres show that the amorphous hump of the polymer predominates, with the three major crystalline peaks of the drug still observed. This may indicate that there was no change in the drug crystalline form during the preparation of the microspheres.

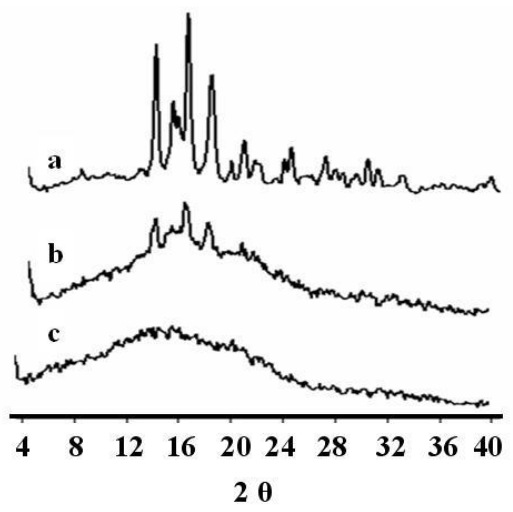

Fig. 8: X-ray diffractograms of: (a) prednisolone, (b) prednisoloneloaded PLA microspheres, and (c) blank PLA microspheres.

The results of the DSC thermograms are in consistence with those obtained from x-ray diffractograms. Figure 9 shows that the drug has a strong endothermic peak at $230^{\circ} \mathrm{C}$ due to the melting of the drug $^{16}$. The smaller endothermic peak at $55.5^{\circ} \mathrm{C}$ corresponds to the glass transition $\left(\mathrm{T}_{\mathrm{g}}\right)$ of PLA blank microspheres. It should be mentioned that the $\mathrm{T}_{\mathrm{g}}$ of the untreated PLA is $32.9^{\circ} \mathrm{C}$ as stated by the provider. Moreover, the melting point peak of the drug appeared very small. This decrease in the peak height may be attributed to the effect of dilution of the drug by the polymer.

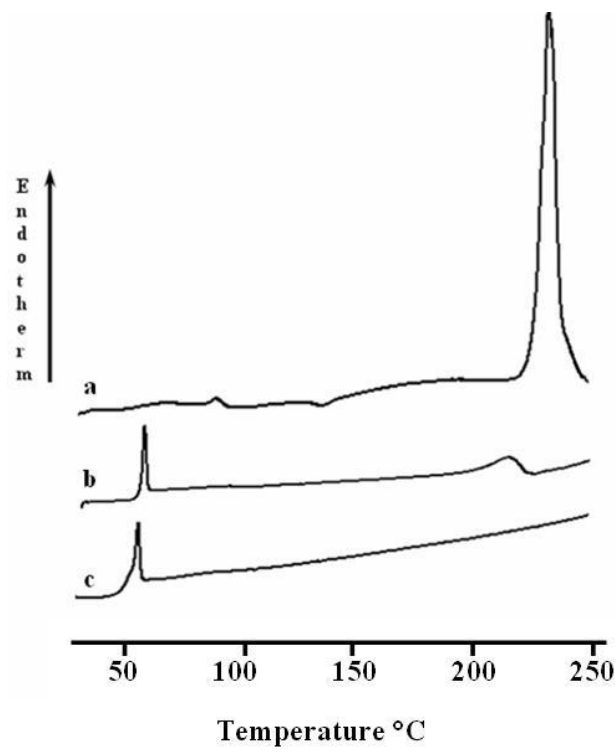

Fig. 9: DSC theromograms of: (a) prednisolone, (b) prednisoloneloaded PLA microspheres, and (c) blank PLA microspheres.

FT-IR spectra show that all the characteristic peaks of the blank microspheres appear clearly in the spectrum of drug-loaded microspheres. The disappearance of the drug absorbance peaks in the drugloaded microspheres spectra can be attributed to the dilution effect (Fig. 10). It can be postulated from these previously mentioned solid-state characterization results that there is no observed interaction between the drug and the polymer, and the drug is present in the form of crystals within 
the polymer matrix. Drug crystals can be observed clearly within the polymer matrix in an SEM image showing a cross-section of a microsphere (not shown). Other authors observed no or negligible drug-PLGA interaction within the prepared microspheres and nanoparticles when drugs with related nuclei were used ${ }^{25 \& 26 .}$

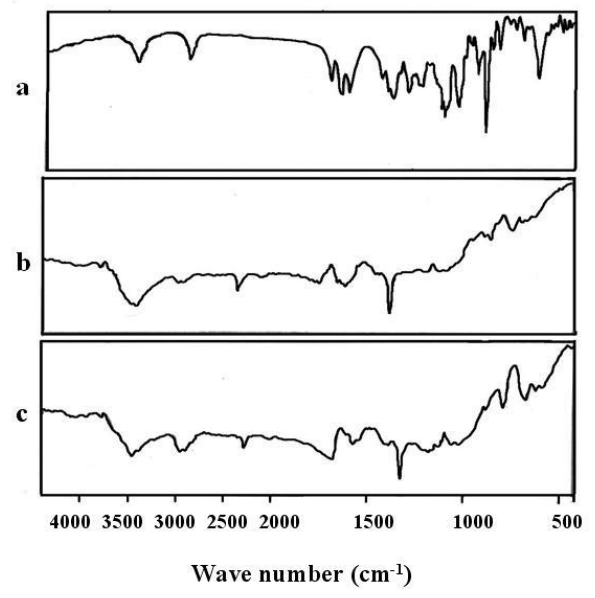

Fig. 10: FT-IR spectra of: (a) prednisolone, (b) prednisoloneloaded PLA microspheres, and (c) blank PLA microspheres.

\section{Kinetics of drug release}

In almost all formulae, drug release from the microspheres is characterized by a biphasic pattern, consisting of an initial rapid rate, followed by a slower release rate. This rapid initial release was attributed to the burst effect due to the presence of the drug onto or near the surface. The rates slowed down afterwards because the drug molecules had to diffuse through channels and pores present in the polymer matrix. As the thickness of the drug-free diffusion layer increased, the difficulty with which the drug diffused through the matrix increased, slowing down the rates of release.

The mechanisms of drug release from the polymeric matrix systems may include diffusion and combined diffusion/matrix erosion. It is believed that the high molecular weight of the used polymer makes the effect of erosion negligible during the period of the release study.

A fundamental equation usually applied to fit release data is the power law, also known as Peppas equation ${ }^{27}$

$$
\frac{M_{t}}{M_{\infty}}=k t^{n}
$$

Where $M_{t}$ is the drug cumulative amount released after time $t, M_{\infty}$ is the drug amount released at infinity, $k$ is an experimental parameter, and $n$ is a coefficient correlated to the release mechanism $^{25}$. The value of $n$ for a spherical matrix is $\leq 0.43$ for quasiFickian (non-steady state) diffusion mechanism, $\geq 0.85$ where the drug rate of release is independent of time (i.e. zero-order kinetics), and $<0.85$ and $>0.43$ for anomalous nonFickian release mechanism.

The $n$ values of fitting the release data to Peppas equation are listed in Table 3. The values of $n$ do not exceed 0.43, which support the conclusion that diffusion mechanism is predominant, and negate the presence of any erosion of the 
polymeric matrix. This conclusion could be emphasized by fitting the release data to the square-root equation ${ }^{28}$, and/or Baker and Lonsdale model ${ }^{29}$.

The general equation that describes the model proposed by Baker and Lonsdale is as follows:

$$
3 / 2\left[1-(1-F)^{2 / 3}\right]-F=k t
$$

Where $F$ is the fraction of the drug released, $t$ is the time, and $k$ is the specific rate constant. So that, upon plotting $3 / 2\left[1-(1-F)^{2 / 3}\right]-F$ versus time for a drug that diffuses from a spherical polymeric matrix, a linear relationship is expected ${ }^{30 \& 31}$.

According to Seki et al., the value of $k$ should be directly proportional to the initial drug loading in the PLA microspheres when the drug is released by diffusion through the capillaries or a series of pores in the PLA microspheres ${ }^{30}$.

A comparison between determination coefficients $\left(r^{2}\right)$ of formulae F\#6, 3, 7, and 8 (drug:polymer ratios of $1: 6,1: 4,1: 3$, and $1: 2$, respectively) after fitting their release data to Baker and Lonsdale model (Table 3) show that the values of $\left(r^{2}\right)$ increase in a direct proportion to drug:polymer ratio. This observation can be indicative of the mechanism of drug diffusion from the microspheres, which is believed to be through capillaries or/and a series of pores in the polymeric matrix.

The results of fitting the release data to the planar model (Higuchi model) and to the spherical model (Baker and Lonsdale model) showed that the determination coefficients for most of the formulae have greater values in Higuchi model, rather than in Baker and Lonsdale model. The exception was formula F\#8 (with actual drug loading of about $32.4 \%$ w/w) (Table 3). it should be mentioned that formula F\#8 is the one which gave the highest rate and extent of release among all additive-free formulae (about 73\% of the encapsulated drug along 96 hours). As mentioned before, the high actual drug content in formula F\#8 helped the continual formation of pores as more drug is released. From the prementioned explanation, It can be suggested that the radius of the receding spherical matrix inside the microsphere (which contains insoluble drug) is decreasing significantly. This pronounced decrease in the radius made the microspheres from $\mathrm{F} \# 8$ to be much closer to Baker-Lonsdale spherical matrix.

Other formulae showed much less extent and/or rate of drug released, when compared to F\#8. This may indicate that the radii of the receding spherical matrices inside these microspheres were decreasing, but to a lesser extent. As a result, the surface

Table 3:Fitting of the release data, up to 48 hours, to various mathematical models.

\begin{tabular}{|c|c|c|c|}
\hline F\# & Peppas model & $\begin{array}{c}\text { planar matrix } \\
\text { (Higuchi model) }\end{array}$ & $\begin{array}{c}\text { spherical matrix } \\
\text { (Baker-Lonsdale }\end{array}$ \\
\hline
\end{tabular}




\begin{tabular}{|c|c|c|c|c|c|c|}
\hline & & & & \multicolumn{2}{|c|}{ model $)$} \\
\hline & $\mathrm{r}^{2}$ & $\mathrm{n}$ & $\mathrm{r}^{2}$ & $\mathrm{k}\left(\mathrm{hr}^{-0.5}\right)$ & $\mathrm{r}^{2}$ & $\mathrm{k}\left(\mathrm{hr}^{-1}\right)$ \\
\hline 1 & 0.9775 & 0.1501 & 0.9241 & 0.0428 & 0.8774 & 0.0009 \\
\hline 2 & 0.9916 & 0.221 & 0.9613 & 0.0448 & 0.9325 & 0.0007 \\
\hline 3 & 0.9935 & 0.2676 & 0.9631 & 0.043 & 0.9507 & 0.0005 \\
\hline 4 & 0.9842 & 0.1634 & 0.9438 & 0.0325 & 0.9155 & 0.0004 \\
\hline 5 & 0.9561 & 0.1477 & 0.8878 & 0.0372 & 0.8313 & 0.0007 \\
\hline 6 & 0.9817 & 0.3072 & 0.945 & 0.0245 & 0.8792 & 0.0002 \\
\hline 7 & 0.9852 & 0.2275 & 0.9606 & 0.0467 & 0.9548 & 0.0007 \\
\hline 8 & 0.996 & 0.3028 & 0.9886 & 0.0722 & 0.9939 & 0.0016 \\
\hline 9 & 0.9994 & 0.4207 & 0.9993 & 0.0492 & 0.9988 & 0.0005 \\
\hline 10 & 0.9612 & 0.4211 & 0.9265 & 0.0334 & 0.897 & 0.0002 \\
\hline
\end{tabular}

areas of the release decrease only slightly. This observation can also occur when most of the released fractions were located near to the surface of the microspheres. When these fractions are released, no significant changes in the radii of the receding spheres occurred, resulting in a better fitting to the Higuchi's planar model.

In conclusion, the impact of the formulation parameters on the EE\% and release mechanism of the prepared and characterized microsphres was evaluated. This study paved the route for the formulator to determine various parameters in order to obtain the sought release pattern. From the study, it could be suggested that Formula F\#8 particularly seems to be the best because it showed high
EE\% (97\%) and continued steady drug release for 4 days.

\section{Acknowledgement}

The authors would like to thank professor Ragai K. Ibrahim (Concordia University, Quebec, Canada) for providing the PLA, and professor Achim Goepferich (University of Regensburg, Regensburg, Germany) for giving access to the particle size analysis instrument used in this study.

\section{REFERENCES}

1- T. Hickey, D. Kreutzer, D. Burgess and F. Moussy, Biomaterials, 23, 1649 (2002).

2- S. Bozdag, S. Calis, H. S. Kas, M. T. Ercan, I. Perkosy and A. A. Hincal, J. Microencapsul., 18, 443 (2001). 
3- H. K. Kim and T. G. Park, J. Control. Release, 98, 115 (2004).

4- F. Tewes, E. Munnier, B. Antoon, L. Ngaboni Okassa, S. Cohen-Jonathan, H. Marchais, L. Douziech-Eyrolles, M. Souce, P. Dubois and I. Chourpa, Eur. J. Pharm. Biopharm., 66, 488 (2007).

5- J. Herrmann and R. Bodmeier, ibid., 45, 75 (1998).

6- M. Ricci, P. Blasi, S. Giovagnoli, C. Rossi, G. Macchiarulo, G. Luca and G. Basta, R. Calafiore. 2005, J. Control. Release, 107, 395 (2005).

7- A. Hincal, S. Calis, Microsphere preparation by solvent evaporation method. In: D. Wise (Ed), Handbook of Pharmaceutical Controlled Release Technology. New York, Marcel Dekker Inc., 2000, p. 329.

8- L. Brannon-Peppas and M. Vert, Polylactic and polyglycolic acids as drug delivery carriers, ibid., $p$. 99.

9- J. P. Benoit, F. Courteille and C. Thies., Int. J. Pharm., 29, 95 (1986).

10- S. Benita, J. P. Benoit, F. Puisieux and C. Thies., J. Pharm. Sci., 73, 1721 (1984).

11- H. Eroglu, H. S. Kas, L. Oner, O. F. Turkoglu, N. Akalan, M. F. Sargon and N. Ozer, J. Microencapsul., 18, 603 (2001).

12- B. S. Zolnik, P. E. Leary and D. J. Burgess, J. Control. Release, 112, 293 (2006).

13- K. Parfitt, S. C. Sweetman, P. S. Blake, A. V. Pearson, Martindale, The Complete Drug Reference, $32^{\text {nd }}$ ed. London, UK:
The Pharmaceutical Press, 1999 p. 1048.

14- G. P. Chrousos and A. N. Margioris. Adrenocorticosteroids and adrenocortical antagonists. In: B. G. Katzung, (Ed.). Basic and Clinical Pharmacology, $8^{\text {th }}$ Ed., Mcgraw Hill (2001).

15- E. Esposito, E. Menegatti and R. Cortesi. Int. J. Pharm., 288, 35 (2005).

16- S. L. Ali, Prednisolone. In: H. G. Brittain, (Ed.) Analytical Profiles of Drug Substances and excipients, Vol. 21. London, UK: Pharmaceutical Press, 1992, p. 415.

17- H. Jeffery, S. S. Davis and D. T. O'Hagan, Int. J. Pharm., 77, 169 (1991).

18- X. Liu, Ph.D Thesis, Drexel University, USA (2003).

19- G. Trapani, A. Lopedota, G. Boghetich, A. Latrofa, M. Franco, E. Sanna and G. Liso, Int. J. Pharm., 268, 47 (2003).

20- A. Budhian, S. J. Siegel and K. L. Winey, Int. J. Pharm., 336, 375 (2007).

21- P. C. Chen, Y. J. Park, L. C. Chang, D. S. Kohane, R. H. Bartlett, R. Langer, V. C. Yang and J. Biomed. Mater. Res., 70A, 412 (2004).

22- M. Cavalier, J. P. Benoit and C. Thies, J. Pharm. Pharmacol., 38, 249 (1986).

23- R. Bodmeier and J. W. Mcginity, Pharm. Res., 4, 465 (1987).

24- J. K. Lalla and K. Sapna, 1993. J. Microencapsul., 10, 449 (1993).

25- S. Giovagnoli, P. Blasi, M. Ricci, A. Schoubben, L. Perioli and C. 
Rossi, J. Pharm. Sci., 97, 303 (2008).

26- C. Gomez-Gaete, N. Tsapis, M. Besnard, A. Bochet and E. Fattal, Int. J. Pharm., 331, 153 (2007).
27- P. L. Ritger and N. A. Peppas, J. Control. Release, 5, 23 (1987).

28- T. Higuchi, J. Pharm. Sci., 51, 802 (1963).

29- R. W. Baker and H. K. Lonsdale, In: A. C. Tanquary, R. E. Lacey Ed., Controlled release of biological active agents. New York: Plenum., 1974, p. 15.

30- T. Seki, T. Kawaguchi, H. Endoh, K. Ishikawa, K. Juni and M. Nakano, J. Pharm. Sci., 79, 985 (1990).

31- L. Fang-I, J. H. Kuo, K. C. Sung and O. Y. P. Hu, Int. J. Pharm., 257, 23 (2003). 